Sargon Ziyeh

Ansgar Berlis

Rudolf Korinthenberg

Joachim Spreer

Martin Schumacher

\section{Selective involvement of the globus pallidus and dentate nucleus in succinic semialdehyde dehydrogenase deficiency}

\begin{abstract}
MRI findings in a 12year-old boy with succinic semialdehyde dehydrogenase (SSADH) deficiency are described. SSADH deficiency is a rare neurometabolic disorder of GABA catabolism. The clinical diagnosis is difficult and the disease is underdiagnosed. MRI showed an unusual pattern with hyperintense signal in the globus pallidus and cerebellar dentate nucleus in T2-weighted images. The remaining basal ganglia and white matter were normal. This is the second report showing this particular pattern of pallidal-dentate nucleus involvement, which might
\end{abstract}

be suggestive for SSADH deficiency.
S. Ziyeh $(\bowtie) \cdot$ A. Berlis $\cdot$ J. Spreer M. Schumacher

Department of Neurosurgery,

University of Freiburg, Germany

E-mail: ziyeh@nz.ukl.uni-freiburg.de

Fax: + 49-761-2705171

S. Ziyeh

Section of Neuroradiology, Neurozentrum, Breisacherstr. 64, 79106 Freiburg, Germany

R. Korinthenberg

Department of Neuropaediatrics,

University of Freiburg, Germany

\section{Introduction}

Succinic semialdehyde dehydrogenase (SSADH) deficiency is a rare neurometabolic disorder of GABA catabolism with autosomal recessive inheritance. GABA is a major central nervous system inhibitory neurotransmitter. Approximately 150 patients have been identified world-wide [1]. Deficient oxidation of the intermediate metabolite succinic semialdehyde leads to accumulation of GABA and 4-hydroxybutyric acid (GHB). GHB possesses a variety of neuromodulatory functions. Both GABA and GHB are assumed to contribute to the pathogenesis of the disorder [1]. The biochemical key finding is elevated GHB in urine and plasma of affected patients. SSADH assay in white blood cells offers rapid confirmation of SSADH deficiency.

Patients with SSADH deficiency present with motor, mental and language delay, hypotonia, ataxia, behavioural problems, hyperkinesis, seizures, neonatal problems and EEG abnormalities [2]. These are nonspecific signs of childhood encephalopathy. SSADH deficiency usually does not show symptoms suggestive of an inborn error of metabolism, such as hyperammonaemia, hypoglycaemia, episodic decompensation, vomiting and metabolic acidosis. The clinical phenotype of SSADH deficiency is extremely heterogeneous, even within families. Consequently, the diagnosis may be delayed or even missed [2].

We report the MRI findings in a 12-year-old boy with SSADH deficiency. The possible role of MRI in the diagnosis of SSADH deficiency will be discussed.

\section{Case report}

The 12-year-old boy was the child of non-consanguineous parents. His development was normal during the first 2 years of life. Later, he developed severe language delay and never exceeded three-word sentences. His motor development was normal except for some 
fine-motor deficits and slight ataxia. He was hyperkinetic and unable to concentrate for more than $10 \mathrm{~min}$.

Urine gas chromatography-spectrometry showed increased GHB concentration. The diagnosis was confirmed by decreased SSADH activity in cultured lymphoblasts. Initiation of standarddose vigabatrin therapy improved concentration, social interaction and hyperkinesia, but the dose had to be reduced because of induction of generalised tonic-clonic seizures. The clinical and therapeutic aspects of this case are described in detail in a previous report [3].

MRI was performed on a 2.0-T Bruker MEDSPEC scanner. Axial T1-weighted (T1-W) spin-echo and T2-weighted (T2-W) RARE sequences (TR/TE 2,700/20, $6 \mathrm{~mm}$ slice thickness, 1 average) in the axial and coronal planes were acquired. No contrast medium was given.

MRI showed bilateral oval lesions with hyperintense signal in the dorsolateral portion of the globus pallidus on T2-W images. These hyperintensities were surrounded by a subtle hypointense ring. The rostral and medial part of the globus pallidus appeared normal (Fig. 1). Additionally, T2-W images showed symmetrically increased signal of the cerebellar dentate nucleus (Fig. 2). No other grey-matter involvement was noted. White-matter changes were lacking. T1-W images only showed slightly hypointense signal in the globus pallidus.

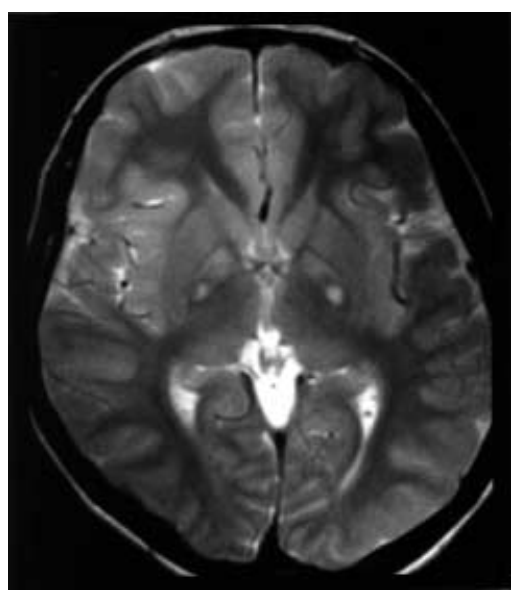

Fig. 1. Axial T2-W MRI shows bilateral oval lesions with hyperintense signal in the dorsolateral globus pallidus, surrounded by a subtle hypointense rim

\section{Discussion}

The diagnosis of SSADH deficiency may be hampered owing to non-specific and variable presenting symptoms. Neuroimaging findings are also generally considered to be rather non-specific. Cerebral atrophy and hyperintense signal in the globus pallidus on MRI, either isolated or in combination with thalamic lesions, have been reported $[2,4]$. A recent case report showed cerebellar vermian atrophy and subtle supratentorial white-matter changes [5]. However, no systematic MRI studies exist for this rare disorder.

It is important to establish the diagnosis of SSADH as early as possible and parents should receive genetic counselling. In some patients, treatment with vigabatrin (gamma-vinyl GABA) leads to clinical improvement. Vigabatrin acts as an irreversible inhibitor of GABAtransaminase, which is the enzyme that converts GABA into succinic semialdehyde [1].

In our patient we observed a particular pattern of enhanced signal in $\mathrm{T} 2-\mathrm{W}$ images with symmetrical involvement of the globus pallidus and cerebellar dentate nucleus. White-matter changes were absent. It is noteworthy that identical MRI findings in an 11-year-old Turkish boy were reported in a recent paper by Yalcinkaya et al. [6]. Isolated pallidal signal hyperintensities on $\mathrm{T} 2-\mathrm{W}$ images may be observed in methylmalonic aciduria, in carbon monoxide poisoning and kernicterus [7]. Abnormal signal of the dentate nucleus may be the leading MRI feature in infantile Refsum's disease [8] and atypical cases of Wilson's disease [9]. However, the clinical phenotype in these disorders is different from SSADH deficiency.

The subtle hypointense rim surrounding the pallidal hyperintensities in our patient created an 'eye-of-thetiger' sign, which is the hallmark in Hallervorden-Spatz disease. However, in Hallervorden-Spatz disease marked hypointensities on $\mathrm{T} 2-\mathrm{W}$ images affect the entire globus pallidus [10]. In our case they were only focal.
Fig. 2a,b. T2-W MRI. a Axial and $\mathbf{b}$ coronal images showing bilateral increased signal in the cerebellar dentate nucleus
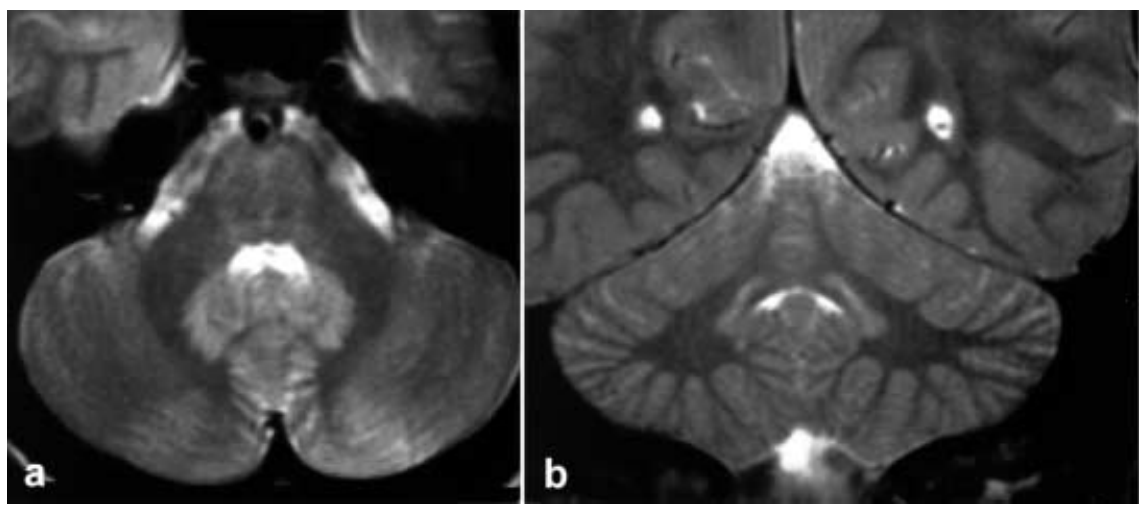
In L-2-OH-glutaric-aciduria, both globus pallidus and dentate nucleus show increased signal on $\mathrm{T} 2-\mathrm{W}$ images. However, white-matter abnormalities seem to be present in virtually all patients, and putamen and caudate head are often affected in addition [7]. In Canavan's disease, leucoencephalopathy is the predominant feature, but globus pallidus and dentate nucleus may additionally be affected [7]. Bilateral signal abnormalities in the globus pallidus and dentate nucleus are common findings in cerebrotendinous xanthomatosis. MRI constantly demonstrates periventricular whitematter changes in all patients, and the disorder shows a distinct clinical pattern with bilateral cataracts, diarrhoea, various neurological symptoms and tendon xanthomas [11].

It seems that the combination of isolated $\mathrm{T} 2$ hyperintense changes in the globus pallidus and dentate nucleus is rather uncommon in toxic, hypoxic and metabolic diseases. Together with the report of Yalcinkaya et al. [6], our case indicates that in the appropriate clinical context this pattern might be suggestive for SSADH deficiency.

In conclusion, in a retarded child the isolated pallidal-dentate MRI pattern with absent white-matter changes should raise the suspicion of SSADH deficiency and prompt biochemical analysis of GHB.

\section{References}

1. Gibson KM, Hoffmann GF, Hodson AK, et al (1998) 4-hydroxybutyric acid and the clinical phenotype of succinic semialdehyde dehydrogenase deficiency, an inborn error of GABA metabolism. Neuropediatrics 29:14-22

2. Gibson KM, Christensen E, Jakobs C, et al (1997) The clinical phenotype of succinic semialdehyde dehydrogenase deficiency (4-hydroxybutyric aciduria): case reports of 23 new patients. Pediatrics 99:567-574

3. Matern D, Lehnert W, Gibson KM, et al (1996) Seizures in a boy with succinic semialdehyde dehydrogenase deficiency treated with vigabatrin (gamma-vinylGABA). J Inher Metab Dis 19:313-318
4. Uziel G, Bardelli P, Pantaleoni C, et al (1993) 4-Hydroxybutyric aciduria: clinical findings and vigabatrin therapy. J Inher Metab Dis 16:520-522

5. AI-Essa MA, Bakheet SM, Patay ZJ, et al (2000) Clinical, fluorine-18 labeled 2-fluoro-2-deoxyglucose positron emission tomography (FDG PET), MRI of the brain and biochemical observations in a patient with 4-hydroxybutyric aciduria; a progressive neurometabolic disease. Brain Dev 22:127-131

6. Yalcinkaya C, Gibson KM, Gunduz E, et al (2000) MRI findings in succinic semialdehyde dehydrogenase deficiency. Neuropediatrics 31:45-46

7. Barkovich AJ (2000) Pedatric neuroimaging, 3rd edn. Lippincott Williams \& Wilkins, Philadelphia

8. Dubois J, Sebag G, Argyropoulou M, et al (1991) MR findings in infantile Refsum disease: case report of two family members. AJNR 12:1159-1160
9. Matsuura T, Sasaki H, Tashiro K (1998) Atypical MR findings in Wilson's disease: pronounced lesions in the dentate nucleus causing tremor. J Neurol Neurosurg Psych 64:161

10. Guillerman RP (2000) The eye-ofthe-tiger sign. Radiology 217:895-896

11. Barkhof F, Verrips A, Wesseling P, et al (2000) Cerebrotendinous xanthomatosis: the spectrum of imaging findings and the correlation with neuropathologic findings. Radiology 217:869-876 